\title{
The imported infections among foreign travelers in China: A descriptive study Running title: Imported infections in China
}

\section{Qiang Xu}

Academy of Military Medical Sciences Institute of Microbiology and Epidemiology

\section{Zhi-Wei Li}

Capital Medical University

Xiao-Ai Zhang

Beijing Institute of Microbiology and Epidemiology

Meng-Yang Liu

Capital Medical University

Jin-Long Wang

Chinese Center for Disease Control and Prevention

\section{Hai-Yang Zhang}

Beijing Institute of Microbiology and Epidemiology

\section{Li-Ping Wang}

Chinese Center for Disease Control and Prevention

\section{Xiu-Hua Guo}

Capital Medical University

\section{Li-Qun Fang}

Beijing Institute of Microbiology and Epidemiology

\section{Wei Liu ( $\boldsymbol{\nabla}$ Iwbime@163.com )}

Beijing Institute of Microbiology and Epidemiology https://orcid.org/0000-0002-9302-8170

\section{Research}

Keywords: Imported infectious diseases, Foreign travelers, Epidemiological characteristics, China

Posted Date: February 8th, 2022

DOI: https://doi.org/10.21203/rs.3.rs-1268969/v1

License: () (i) This work is licensed under a Creative Commons Attribution 4.0 International License. Read Full License 


\section{Abstract \\ Background}

Increasing number of travel-related infections were reported in China, but the spectrum, dynamics and disease burden of imported infections among foreign travelers remain vague.

\section{Methods}

We obtained the data on imported infections among foreign travelers from Custom Inbound Screening System (CISS) and the National Notifiable Infectious Disease Reporting System (NNIDRS). All the infections were classified into respiratory, gastrointestinal, vector-borne, blood/sex-transmitted and mucocutaneous diseases. The epidemiological characteristics and disease burden of imported infections among foreign travelers were investigated.

\section{Results}

In total, 17,189 travelers diagnosed with 58 imported infectious diseases were reported from 2014 to 2018, with an overall incidence rate of 122.59 per million. Respiratory infection (7,351 cases, mainly influenza) and Blood/sex-transmitted diseases (6,114 cases mainly Hepatitis B and HIV infection) were the most frequently diagnosed diseases, followed by vector-borne infections (3,128 cases, mainly dengue fever and malaria). The highest case number was imported from Asia and Europe, while the highest incidence rate was from Africa (296.0 per million). When specific diagnosis was compared, both the highest absolute case number and incidence rate were observed for influenza. An obvious seasonal pattern was observed for vector-borne diseases, with the epidemic spanning from July to November. The origin-destination matrices disclosed the movement of imported infection followed specific routes.

\section{Conclusions}

Our study gave a profile of infectious diseases among foreign travelers traveled into China and pinpointed the priority regions, seasons, populations for prevention and control.

\section{Introduction}

Before the COVID-19 epidemic, international travel had persistently increased in frequency, with the imported infectious diseases causing an increasingly serious public health problem faced by almost all countries. Foreign travelers or immigrants account for an essential proportion of all travelers, with a high risk of spreading infectious diseases across borders by which pathogens can reach new human populations or animal communities. Particularly for the vector borne disease, the movement of patients beyond endemic countries has threatened long-term eradication goals, such as malaria(1). Moreover, the imported cases continue to pose challenges for diagnosis and management for physicians in non-endemic areas, where it 
can be difficult to treat and result in high mortality. Health care providers need a complete understanding of the spectrum of imported infectious diseases and their epidemic patterns, which is critical in containing the potential epidemic or pandemic caused by imported diseases.

In China, the number of foreign travelers has steadily increased from 83.44 million person-times in 2000 to 141.20 million person-times in 2018, associated with increased report of imported infections from 5,261 cases in 2014 to 10,157 in 2016 and 14,700 in 2018(2,3). The imported infection was mainly captured by the costume entrance quarantine and national surveillance system on travel related disease(2-4), which had provided valuable information about the composition, and trends of imported infection. In the current study, we made special efforts on the foreign travelers (here referred to as those with foreign citizenship) to explore the pathogen spectrum and relate the spectrum to their epidemiological features.

\section{Material And Methods}

\section{Data collection}

Full details of obtaining the data on international imported infections had been provided in a previous study (4). Briefly, data were extracted from two anonymous and delinked databases: the Custom Inbound Screening System (CISS) that had covered all 272 entry-exit ports in China (Figure S1), and the National Notifiable Infectious Disease Reporting System (NNIDRS) of Chinese Center for Diseases Control and Prevention (CDC) which covered all 31 provinces in the mainland of China. For diagnosis of specific disease, SOP released by the General Administration of Quality Supervision, Inspection and Quarantine of the People's Republic of China (AQSIQ) was followed that covered all processes of sample collection and laboratory tests (Figure S2) (2). For the screened patients, a standard questionnaire interview was applied to record the clinical and epidemiological data that were related to the travel illness (Figure S3). A predefined standard dataset was used to extract the data from the patient's medical records, including the epidemiological data that were related to the travel history, and clinical data that supported the diagnosis. For the purpose of calculating the incidence rate, the inbound arrivals data in the mainland of China were obtained from the Annual Report of China Tourism Statistics 2015 to 2019. The number of foreign travelers was estimated by six continents, 23 selected countries, travelling year and month from 2014 to 2018 .

\section{Definitions and classifications of imported infections}

Five syndromic diseases were classified based on 67 infectious diseases that were screened from two reporting systems, including respiratory, gastrointestinal, vector-borne disease, blood/sex-transmitted disease and mucocutaneous disease as previously described (2).

Six possible travel purpose designations were used in this analysis: tourism; labour, business; research or student; visiting friends and relatives, etc. Travelers were grouped into six geographic areas according to their originating regions: Africa, Asia, Europe, Latin America, North America, and Oceania.

\section{Statistical analysis}


Descriptive statistics were performed for all variables. Continuous variables were summarized as median and range. Proportions were calculated regarding various disease categories, which were compared for the difference among subgroups by Chi-square test. Incidence rates were calculated as the case number divided by the total number of arriving foreign travelers. We standardized the incidence rate by inbound travel number for each infectious disease, determined the percentage rank, and further represented data as thermodynamic diagrams as previously described $(5,6)$.

For each reported infectious disease, we aggregated the annual mean case numbers exported to each province by the source countries/continents, and further constructed origin-destination matrices. The SAS software (version 9.4) was used for data extraction, sorting and cleaning, and R project (version 3.6.3) and ArcGIS software (version 10.5) were used for graphics presentation and further data analysis.

\section{Results}

\section{Demographic characteristics and infection types of the imported diseases}

During 2014-2018, a total of 17,189 travelers with 58 imported infectious diseases were confirmed out of a totally 140,210.3 thousand person-times arrivers, with an overall incidence rate of 122.59 per million, which comprised of 14,452 cases reported from CISS and 2,737 reported from NNIDRS (Table 1). Travelers of male gender, aged 25-44 years, those originated from Asian countries have accounted for a higher proportion of travel related cases, which were identified from both in-bound custom screening and NNIDRS.

The highest incidence of infection was for respiratory infection (RI), which showed ascending tendency over the 5 study years at an average rate of 24.96 per million (Figure 1A). Influenza was most frequently seen among all types of tested respiratory diseases, with an extraordinarily high level in the year of 2017 and 2018 (Figure 1B). Blood/sex-transmitted infection (BSTI) was the second frequent disease with a consistently increasing incidence from 18.10 per million in 2014 to 70.13 per million in 2018. HIV infection, syphilis, hepatitis $B$ and hepatitis $C$ were among the most frequent diagnosis, with incidence rates of 14.12 , $5.89,18.92$ and 4.48 per million, respectively (Figure 1B). When data from two reporting systems were separately analyzed, respiratory infection and vector-borne diseases were the most common syndrome classification from CISS and NNIDRS, respectively (Figure S4).

\section{The spectrum of imported infection}

Totally 58 imported infectious diseases were diagnosed among 17,189 travelers (Figure 3). The 10 most commonly infections were influenza (proportion of $36.96 \%$ ), hepatitis B $(15.43 \%)$, dengue fever $(13.09 \%)$, HIV (11.51\%), malaria (4.86\%), syphilis (4.81\%), hepatitis C (3.66\%), rhinovirus infection (1.33\%), and tuberculosis $(1.07 \%)$, and norovirus infection $(1.01 \%)$, all collectively taking $93.72 \%(16,110 / 17,189)$ of the confirmed diagnosis. Their annual incidence kept increasing during the study period, especially for HIV infection, hepatitis B and norovirus infection (Figure 2A).

\section{The epidemiological features of imported infections}


The spectrum of illnesses seen among these travelers varied according to age, their travel purpose and source country. Respiratory illnesses and gastrointestinal illnesses were more likely to occur in tourism travelers, vector-borne diseases were more likely to occur in laboring travelers, while mucocutaneous (particularly acute hemorrhagic conjunctivitis and EB virus infection) diseases were more likely to occur in travelers with business purpose (Table S1). There was no difference between genders for the infection spectrum.

By pooling the five-year data, we observed an obvious seasonal pattern, with a high incidence rate of all types of infection plotted in July $(245.00,6.67,40.58,95.8$ and 7.54 per million for respiratory disease, gastrointestinal disease, vector borne disease, blood/sex- transmitted disease and mucocutaneous disease, respectively). This trend was remarkably observed for gastrointestinal disease, mucocutaneous disease and vector borne disease, when compared with other types of infection. (Figure 1C).

\section{The source countries and inbound provinces of imported infections}

Altogether 17,120 ill travelers had source countries recorded. The ill travelers were originated from 152 countries on six continents, with their destination distributed in all 31 provinces in the mainland of China (Figure 4A and 4B). Asian travelers had taken the predominant part of imported cases, accounting for $59.02 \%(10105 / 17120)$ of all imported cases, followed by those from European and African countries. Within Asian countries, Korean travelers had contributed the highest number of cases with RIs, BSTIs and VBDs. The destination provinces which had reported more than 1000 imported cases included Yunnan ( 3,134 cases), Guangdong (2,017 cases), Shanghai (1,939 cases), Inner Mongolia (1,447 cases) and Jiangsu $(1,348$ cases $)$.

The movement of imported infection followed specific routes (Figure 4), in that the connections were more frequently seen between African and Hubei, Guizhou and Jiangxi province (13 cases on average per year), and between Asian (mainly including Burma, Vietnam and Laos) and Yunnan province (592 cases on average per year,) and between Asian (Mongolia and Russia) and Inner Mongolia (233 cases on average per year). Notably, the imported patients in metropolises such as Beijing, Shanghai and Guangdong Provinces tended to be multi-continental sources (Figure 4B).

The annual entry population data were available from six continents and 23 countries, which were used as denominator for incidence analysis. This had accounted for $79.25 \%$ (111.123/140.21 million person-times) of total entry population and $45.96 \%(7,900 / 17,189)$ of total imported cases. When in relate to the source regions, the highest case incidence was reported from the African travelers (296.0 per million), in contrast with the highest case number from Asia, while the lowest incidence was reported from European countries (41.7 per million). When specific diagnosis was compared, both the absolute case number and incidence rate was observed for influenza (Figure 4).

\section{Discussion}

In China, imported infectious diseases remain the major cause of morbidity and mortality despite substantial progress in their control, partially owing to the close connection of China with the rest of the 
world through travel and trade. The previous publications that were built on the real-time monitoring system from enter-exit port have provided a snapshot of travel relate infection over geographic area, or time frame, however, rarely differentiate between native citizens and foreigner travelers for their role in spreading the imported infectious diseases.

In this study, rather than study all entering foreign travelers, we focused on the foreign travelers for analysis, compared the imported notifiable diseases by their travel related data to identify countries, seasons and travel modes that were associated with a high incidence of imported infections. Due to the geographical proximity and frequent international exchanges, Asian travelers contributed the highest number of incoming travelers and cases with imported infectious diseases, however, African travelers had the highest incidence of imported infection, thus posing as the high priority in inbounding quarantine. The imported patients were primarily originated from bordering countries of China, with a high possibility of causing local epidemics (7), such as importation of dengue from Vietnam, Laos and Myanmar into Yunnan, and blood/sextransmitted diseases from Mongolia and Russia into Inner Mongolia, respectively. In contrast, the imported patients reported in metropolis or provinces such as Beijing, Shanghai and Guangdong Provinces, a wide range of source countries that contributed to the imported infections were identified, due to a highly complicated transportation exchange around the world. These features might assist in providing medical recommendations to foreign travelers.

The spectrum of imported infectious diseases from foreign travelers also varied depending on the travel season and the travel purposes. Except for vector-borne diseases that were extraordinarily overrepresented between July and November, all other infectious diseases were evenly detected throughout the year, composing a persistent burden to the medical quarantine work. The major epidemic peak of VBD in travelers might reflect the epidemic pattern of the disease in the original endemic areas. For example, the peaking season of VBD in southeast Asia spanned from June to September, which coincided with those of the imported VBD $(8,9)$. In the case of most important VBDs, dengue cases generally peaked in June and September in Southeast Asian countries $(8,9)$ while malaria cases generally peaked in September to November in African countries (10), which might be responsible for the high and sustained epidemic peak of imported VBDs from July to November. Previous reports on the imported malaria in the UK (11), Finland (12) and Bulgaria (13), and the imported dengue in Japan (14), Spain (15) and East London (16), had revealed the similar epidemic patterns as in the current study. Travelers to dengue-/malaria-endemic countries appear to serve as reliable "sentinels" that may inform the international community of the onset of epidemic activity in specific areas.

During the study period, the incidence of imported infections from foreign travelers had been increasing, especially for gastrointestinal and blood/sex-transmitted diseases. In agreement with previous report that analyzed all the native and foreign cases (2), respiratory infections were the most frequently diagnosis among five infection types. Except for the most common diseases including influenza and rhinovirus infections, the diseases with a small number of imported cases but with epidemic potential and risk of high mortality among humans should also be given prioritized attention, such as MERS (17). Notably, the majority of VBDs were identified by the NNIDS instead of through the custom screening, likely due to the relatively long incubation period of most VBD, which was consistent with a previous study(3). Although 
malaria is no longer endemic in China, the large number and continuous import of malaria have brought great challenges to the goal of a national malaria elimination by 2020 (18). Emerging imported diseases in recent years such as zika and chikungunya also posed serious threats to human health, therefore enhanced screening among foreign travelers should be stressed in the high risky population and seasons as we have determined in the current research.

Our study was subject to several limitations. First, the incidence rate might be underreported for most imported infectious diseases because many patients suffering from post-travel symptoms might be missed from the inbound screening, and not seek medical care due to mild symptoms thereafter, or even if they go to the doctor, they were not judged as travel-related illnesses. Second, we could not calculate the incidence rate of foreign travelers regarding their age, sex or original country since the detailed denominator data were inaccessible. Third, the specific location of the infection was difficult to determine for those travelers who have visited several countries before entering China (We used the last country they visited as the original country). For example, the imported dengue or malaria cases from France, Germany, or Switzerland, where no endemic diseases exist, were quite suspicious.

\section{Conclusions}

In conclusion, our study gave a profile of imported infectious diseases in foreign travelers into China and pinpointed the priority regions, seasons, populations for imported disease prevention and control, which might assist in making medical risk assessments and identifying imported cases in a timely manner, especially those of highly communicable and highly lethal infections.

\section{Abbreviations}

HIV

Human Immunodeficiency Virus

TB

tuberculosis

MERS

Middle East Respiratory Syndrome

ITHC

International Travel Healthcare Center

NNIDRS

National Notifiable Infectious Disease Reporting System

CCDC

Chinese Center for Diseases Control and Prevention

COVID-19

coronavirus disease 2019

\section{Declarations}




\section{Sources of funding}

This work was supported by grants from the China Mega-Project on Infectious Disease Prevention [2018ZX10101003-002, 2017ZX10303401, 2018ZX10713002, and 2018ZX10713001]; the National Natural Science Foundation of China [81825019 and 91846302]; and the National Key Research and Development Project [2019YFC1200604]. The sponsors of the study had no role in study design, data collection, data analysis, interpretation, or writing of the report. The corresponding author had full access to all the data in the study and had final responsibility for the decision to submit for publication.

\section{Acknowledgments}

The data was provided by the General Administration of Customs of the People's Republic of China and Chinese CDC. We thank the healthcare personnel who contributed to the detection, epidemiological investigation, and diagnosis of all suspected infections.

\section{Author contributions}

W.L., L.-Q.F., and X.-H.G. designed the study. Q.X., Z.-W.L., X.-A.Z., M.-Y.L., J.-L.W., H.-Y.Z., and L.-P.W. performed the literature review, data collection and integration. Q.X., Z.-W.L., X.-A.Z., and M.-Y.L., J.-L.W. conducted the analyses under supervision of W.L., L.-Q.F., X.-H.G., H.-Y.Z., and L.-P.W. helped with the analyses. W.L., L.-Q.F., and X.-H.G. wrote the draft of the manuscript. All authors contributed to and approved the final version of the manuscript.

\section{Conflict of Interest}

We declare no competing interests.

-Ethics approval and consent to participate

Not applicable. All patient data were anonymized. This study constituted public health surveillance rather than research in human beings, so ethical approval and consent to participate were not required.

-Consent for publication

Not applicable. This study does not contain data from any individual person.

-Availability of data and material

Raw data are not publicly available and are protected due to data privacy laws, which were used under license for the current study, but are available upon reasonable request to the corresponding author. The request will be responded within one week.

\section{References}


1. Tatem AJ, Jia P, Ordanovich D, Falkner M, Huang Z, Howes R, et al. The geography of imported malaria to non-endemic countries: a meta-analysis of nationally reported statistics. The Lancet Infectious diseases. 2017;17(1):98-107.

2. Fang LQ, Sun Y, Zhao GP, Liu LJ, Jiang ZJ, Fan ZW, et al. Travel-related infections in mainland China, 2014-16: an active surveillance study. The Lancet Public health. 2018;3(8):e385-e94.

3. Wang Y, Wang X, Liu X, Ren R, Zhou L, Li C, et al. Epidemiology of Imported Infectious Diseases, China, 2005-2016. Emerg Infect Dis. 2018;25(1):33-41.

4. Wu Y, Liu MY, Wang JL, Zhang HY, Sun Y, Yuan Y, et al. Epidemiology of imported infectious diseases, China, 2014-18. Journal of travel medicine. 2020;27(8).

5. Yang S, Wu J, Ding C, Cui Y, Zhou Y, Li Y, et al. Epidemiological features of and changes in incidence of infectious diseases in China in the first decade after the SARS outbreak: an observational trend study. The Lancet Infectious diseases. 2017;17(7):716-25.

6. Yu J, Lai S, Geng Q, Ye C, Zhang Z, Zheng Y, et al. Prevalence of rotavirus and rapid changes in circulating rotavirus strains among children with acute diarrhea in China, 2009-2015. J Infect. 2019;78(1):66-74.

7. Hu TS, Zhang HL, Feng Y, Fan JH, Tang T, Liu YH, et al. Epidemiological and molecular characteristics of emergent dengue virus in Yunnan Province near the China-Myanmar-Laos border, 2013-2015. BMC Infect Dis. 2017;17(1):331.

8. Phanitchat T, Zhao B, Haque U, Pientong C, Ekalaksananan T, Aromseree S, et al. Spatial and temporal patterns of dengue incidence in northeastern Thailand 2006-2016. BMC Infect Dis. 2019;19(1):743.

9. Cuong HQ, Vu NT, Cazelles B, Boni MF, Thai KT, Rabaa MA, et al. Spatiotemporal dynamics of dengue epidemics, southern Vietnam. Emerg Infect Dis. 2013;19(6):945-53.

10. Diouf I, Rodriguez Fonseca B, Caminade C, Thiaw WM, Deme A, Morse AP, et al. Climate Variability and Malaria over West Africa. Am J Trop Med Hyg. 2020;102(5):1037-47.

11. Nabarro LEB, Nolder D, Broderick C, Nadjm B, Smith V, Blaze M, et al. Geographical and temporal trends and seasonal relapse in Plasmodium ovale spp. and Plasmodium malariae infections imported to the UK between 1987 and 2015. BMC Med. 2018;16(1):218.

12. Guedes S, Siikamäki H, Kantele A, Lyytikäinen O. Imported malaria in Finland 1995 to 2008: an overview of surveillance, travel trends, and antimalarial drug sales. Journal of travel medicine. 2010;17(6):4004.

13. Rainova IG, Harizanov RN, Kaftandjiev IT, Mikov OD, Tsvetkova ND. Imported malaria in Bulgaria, status and prognosis after eradication in 1965. J Infect Public Health. 2018;11(4):534-9.

14. Fukusumi M, Arashiro T, Arima Y, Matsui T, Shimada T, Kinoshita H, et al. Dengue Sentinel Traveler Surveillance: Monthly and Yearly Notification Trends among Japanese Travelers, 2006-2014. PLoS Negl Trop Dis. 2016;10(8):e0004924.

15. Redondo-Bravo L, Ruiz-Huerta C, Gomez-Barroso D, Sierra-Moros MJ, Benito A, Herrador Z. Imported dengue in Spain: a nationwide analysis with predictive time series analyses. Journal of travel medicine. 2019;26(8). 
16. Riddell A, Babiker ZO. Imported dengue fever in East London: a 6-year retrospective observational study. Journal of travel medicine. 2017;24(3).

17. Wu J, Yi L, Zou L, Zhong H, Liang L, Song T, et al. Imported case of MERS-CoV infection identified in China, May 2015: detection and lesson learned. Euro surveillance: bulletin Europeen sur les maladies transmissibles = European communicable disease bulletin. 2015;20(24).

18. China NHCo. Beijing: Action plan of China malaria elimination (2010-2020). 2015 http://wwwnhcgovcn/jkj/s5873/201005/f84f1c4b0f32420990d23b65a88e2d87shtml.

\section{Table}

Table 1. The characteristics of imported cases among foreign travelers in Chinese mainland, 2014-2018. 
Overall Respiratory Gastrointestinal $(n=17189)$

$(n=7351)$

$(n=282)$

VBD

$(n=3128)$

BSTI

$(n=6114)$

Mucocutaneous

$(n=314)$

Sex

\begin{tabular}{lllllll} 
Male & $\begin{array}{l}12347 \\
(71.8)\end{array}$ & $\begin{array}{l}5769 \\
(78.5)\end{array}$ & $165(58.5)$ & $\begin{array}{l}1861 \\
(59.5)\end{array}$ & $\begin{array}{l}4310 \\
(70.5)\end{array}$ & $242(77.1)$ \\
\hline \multirow{2}{*}{ Female } & 4842 & 1582 & $117(41.5)$ & 1267 & 1804 & $72(22.9)$ \\
& $(28.2)$ & $(21.5)$ & & $(40.5)$ & $(29.5)$ &
\end{tabular}

Age $\quad 33(25-$

\begin{tabular}{|c|c|c|c|c|c|c|}
\hline $0-14$ & $1485(8.6)$ & 932 (12.7) & $30(10.6)$ & $\begin{array}{l}469 \\
(15.0)\end{array}$ & $18(0.3)$ & $36(11.5)$ \\
\hline $15-29$ & $\begin{array}{l}5326 \\
(31.0)\end{array}$ & $\begin{array}{l}1755 \\
(23.9)\end{array}$ & $97(34.4)$ & $\begin{array}{l}1130 \\
(36.1)\end{array}$ & $\begin{array}{l}2258 \\
(36.9)\end{array}$ & $86(27.4)$ \\
\hline $30-44$ & $\begin{array}{l}6040 \\
(35.1)\end{array}$ & $\begin{array}{l}2425 \\
(33.0)\end{array}$ & $68(24.1)$ & $\begin{array}{l}903 \\
(28.9)\end{array}$ & $\begin{array}{l}2540 \\
(41.5)\end{array}$ & $104(33.1)$ \\
\hline $45-59$ & $\begin{array}{l}3172 \\
(18.5)\end{array}$ & $\begin{array}{l}1558 \\
(21.2)\end{array}$ & $33(11.7)$ & $\begin{array}{l}472 \\
(15.1)\end{array}$ & $\begin{array}{l}1050 \\
(17.2)\end{array}$ & $59(18.8)$ \\
\hline$\geq 60$ & $1166(6.8)$ & $681(9.3)$ & $54(19.1)$ & $154(4.9)$ & $248(4.1)$ & $29(9.2)$ \\
\hline
\end{tabular}

Destination

\begin{tabular}{|c|c|c|c|c|c|c|}
\hline Africa & $907(5.3)$ & $137(1.9)$ & $8(2.8)$ & $\begin{array}{l}479 \\
(15.3)\end{array}$ & $282(4.6)$ & $1(0.3)$ \\
\hline Asia & $\begin{array}{l}10105 \\
(58.8)\end{array}$ & $\begin{array}{l}4833 \\
(65.7)\end{array}$ & $188(66.7)$ & $\begin{array}{l}2545 \\
(81.4)\end{array}$ & $\begin{array}{l}2278 \\
(37.3)\end{array}$ & 261 (83. \\
\hline Europe & $1342(7.8)$ & $694(9.4)$ & $21(7.4)$ & $22(0.7)$ & $594(9.7)$ & $11(3.5)$ \\
\hline $\begin{array}{l}\text { Latin } \\
\text { America \& } \\
\text { Caribbean }\end{array}$ & $165(1.0)$ & $94(1.3)$ & $4(1.4)$ & $19(0.6)$ & $42(0.7)$ & $6(1.9)$ \\
\hline $\begin{array}{l}\text { North } \\
\text { America }\end{array}$ & $722(4.2)$ & $520(7.1)$ & 34 (12.1) & $3(0.1)$ & $157(2.6)$ & $8(2.5)$ \\
\hline Oceania & $536(3.1)$ & $468(6.4)$ & $19(6.7)$ & $10(0.3)$ & $26(0.4)$ & $13(4.1)$ \\
\hline unknown & $\begin{array}{l}3412 \\
(19.8)\end{array}$ & $605(8.2)$ & $8(2.8)$ & $50(1.6)$ & $\begin{array}{l}2735 \\
(44.7)\end{array}$ & $14(4.5)$ \\
\hline \multicolumn{7}{|l|}{$\begin{array}{l}\text { Infection } \\
\text { year }\end{array}$} \\
\hline 2014 & $1280(7.4)$ & $445(6.1)$ & $10(3.5)$ & $\begin{array}{l}334 \\
(10.7)\end{array}$ & $477(7.8)$ & $14(4.5)$ \\
\hline 2015 & $\begin{array}{l}2413 \\
(14.0)\end{array}$ & $\begin{array}{l}1025 \\
(13.9)\end{array}$ & $16(5.7)$ & $\begin{array}{l}644 \\
(20.6)\end{array}$ & $\begin{array}{l}693 \\
(11.3)\end{array}$ & 35 (11.1) \\
\hline 2016 & 3545 & 1740 & $\begin{array}{l}63(22.3) \\
\quad \text { Page 11/ }\end{array}$ & 494 & 1177 & 71 (22.6) \\
\hline
\end{tabular}




\begin{tabular}{|c|c|c|c|c|c|c|}
\hline 2017 & $\begin{array}{l}5421 \\
(31.5)\end{array}$ & $\begin{array}{l}2351 \\
(32.0)\end{array}$ & $69(24.5)$ & $\begin{array}{l}1254 \\
(40.1)\end{array}$ & $\begin{array}{l}1625 \\
(26.6)\end{array}$ & $122(38.9)$ \\
\hline 2018 & $\begin{array}{l}4530 \\
(26.4)\end{array}$ & $\begin{array}{l}1790 \\
(24.4)\end{array}$ & $124(44.0)$ & $\begin{array}{l}402 \\
(12.9)\end{array}$ & $\begin{array}{l}2142 \\
(35.0)\end{array}$ & $72(22.9)$ \\
\hline
\end{tabular}

VBD, vector borne disease. BSTI, Blood/sex- transmitted infection

\section{Figures}

\section{Figure 1}

Annual trends and seasonal pattern of imported infections among foreign travelers in the mainland of China

\section{Figure 2}

The spectrum of imported infections in foreign travelers with foreign nationality in the mainland of China, 2014-2018

Notes: Inf: Influenza; Rhl: Rhinovirus infection; Adl: Adenovirus infection; HIl: Haemophilus influenzae infection; RSVI: Respiratory syncytial virus infection; CP: Chicken pox; HMVI: Human metapneumonia virus infection; Cl: Coronavirus infections; PVI: Parainfluenza virus infection; Pnl: Pneumococcal infection; Mea: Measles; EM: Epidemic mumps; MPI: Mycoplasma pneumoniae infection; LD: Legionella disease; SI: Streptococcal infection; BVI: Boka virus infection; CPI: Chlamydia pneumonia infection; Per: Pertussis; IAP: Infectious atypical pneumonia; MERS: Middle East Respiratory Syndrome; TB: Tuberculosis; NI: Norovirus infection; ECl: Escherichia coli infection; VPI: Vibrio parahaemolyticus infection; HA: Hepatitis A; Rol: Rotavirus infection; BD: Bacterial dysentery; TF: Typhoid fever; PF: Paratyphoid fever; HE: Hepatitis E; AD: Amiba's dysentery; Cho: Cholera; PWM: Pin worm disease; Mal: Malaria; DF: Dengue fever; CF: Chikungunya fever; TD: Tsutsugamushi disease; EB: Encephalitis B; Bru: Brucellosis; Rab: Rabies; EIE: Epidemic and endemic typhus; Clo: Clonorchiasis; FE: Forest encephalitis; Syp: Syphilis; NGI: Neisseria gonorrhoeae infection; ClHT: Chlamydia infection of genitourinary tract; Tri: Trichomoniasis; HB: Hepatitis B; HC: Hepatitis C; EBVI: EB virus infection; El: Enterovirus infection; Tra: Trachoma; NT: Neonatal tetanus; Her: Herpes; AHC: Acute hemorrhagic conjunctivitis.

\section{Figure 3}


The standardized incidence rate of imported infection from foreign travelers with foreign nationality in 6 continents and 23 selected countries in China

Notes: Inf: Influenza; Rhl: Rhinovirus infection; Adl: Adenovirus infection; HIl: Haemophilus influenzae infection; RSVI: Respiratory syncytial virus infection; CP: Chicken pox; HMVI: Human metapneumonia virus infection; Cl: Coronavirus infections; PVI: Parainfluenza virus infection; Pnl: Pneumococcal infection; Mea: Measles; EM: Epidemic mumps; MPI: Mycoplasma pneumoniae infection; LD: Legionella disease; SI: Streptococcal infection; BVI: Boka virus infection; CPI: Chlamydia pneumonia infection; Per: Pertussis; IAP: Infectious atypical pneumonia; MERS: Middle East Respiratory Syndrome; TB: Tuberculosis; NI: Norovirus infection; ECl: Escherichia coli infection; VPI: Vibrio parahaemolyticus infection; HA: Hepatitis A; Rol: Rotavirus infection; BD: Bacterial dysentery; TF: Typhoid fever; PF: Paratyphoid fever; HE: Hepatitis E; AD: Amiba's dysentery; Cho: Cholera; PWM: Pin worm disease; Mal: Malaria; DF: Dengue fever; CF: Chikungunya fever; TD: Tsutsugamushi disease; EB: Encephalitis B; Bru: Brucellosis; Rab: Rabies; EIE: Epidemic and endemic typhus; Clo: Clonorchiasis; FE: Forest encephalitis; Syp: Syphilis; NGI: Neisseria gonorrhoeae infection; CIHT: Chlamydia infection of genitourinary tract; Tri: Trichomoniasis; HB: Hepatitis B; HC: Hepatitis C; EBVI: EB virus infection; El: Enterovirus infection; Tra: Trachoma; NT: Neonatal tetanus; Her: Herpes; AHC: Acute hemorrhagic conjunctivitis.

\section{Figure 4}

The originating countries and destiny provinces in China of foreign travelers with imported infections.

\section{Supplementary Files}

This is a list of supplementary files associated with this preprint. Click to download.

- SUPPLEMENTARYsubmitnew.docx 